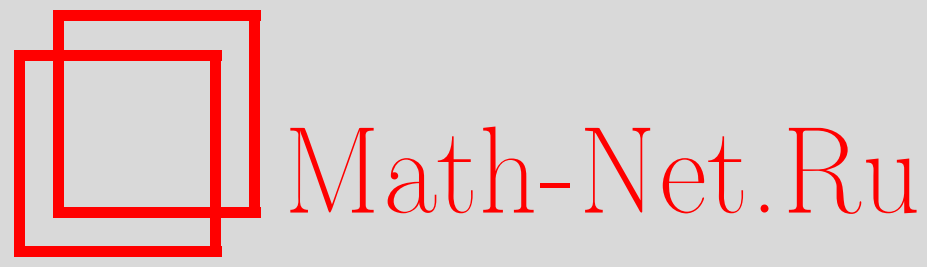

Ц. Чжан, Ц. Шу, Точные условия глобального существования решений квазилинейного уравнения Шрёдингера, Функи. анализ и его прил., 2008, том 42, выпуск 2, 68-74

DOI: https://doi.org/10.4213/faa2903

Использование Общероссийского математического портала MathNet.Ru подразумевает, что вы прочитали и согласны с пользовательским соглашением http://www . mathnet.ru/rus/agreement

Параметры загрузки:

IP : 3.95 .254 .165

26 апреля 2023 г., 14:55:01

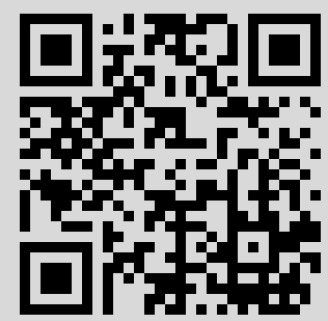


Функиионалъный анализ и его приложения

2008, т. 42, вып. 2, с. 68-74

УДК $517.956+517.958$

\title{
Точные условия глобального существования решений квазилинейного уравнения Шрёдингера*
}

\author{
(c) 2008. Цзянь ЧжАн, Цзи Шу
}

\section{§1. Введение}

Мы рассматриваем квазилинейные уравнения Шрёдингера вида

$$
i \varphi_{t}=-\Delta \varphi+V(x) \varphi-f\left(|\varphi|^{2}\right) \varphi-k \Delta h\left(|\varphi|^{2}\right) h^{\prime}\left(|\varphi|^{2}\right) \varphi,
$$

получающиеся как модели некоторых физических явлений. Здесь $V=V(x)$, $x \in \mathbb{R}^{N}$, - заданный потенциал, $k$ - вещественная постоянная, а $f$ и $h-$ вещественные функции. Например, если $h(s)=s$, то получается уравнение

$$
i \varphi_{t}=-\Delta \varphi+V(x) \varphi-f\left(|\varphi|^{2}\right) \varphi-k \Delta h\left(|\varphi|^{2}\right) \varphi,
$$

которое Курихара [1] (ср. [2], [3]) назвал уравнением сверхтекучей пленки в физике плазмы. При $h(s)=(1+s)^{1 / 2}$ уравнение (1) моделирует автоканализацию сверхмощных ультракоротких лазерных импульсов в веществе, см. [4]-[7] и ссылки в [8]. Уравнение (1) появляется также в физике плазмы и механике жидкостей ([1], [2], [9]-[11]), в теории гейзенберговских ферромагнетиков и магнонов ([12]-[16]), в диссипативной квантовой механике [17] и теории конденсированного состояния вещества [18]. В математической литературе результатов об уравнениях вида (1), (2) очень мало. Локальные и глобальные результаты о корректности задачи Коши приводятся в работе [8] для случая $h(s)=(1+s)^{1 / 2}$ и $N=1,2,3 ;$ случай, когда $h(s)=s$, а $N=1$, изучен в [3], а случай функций $h$ общего вида рассмотрен в [19] для $N=1$ и в [20], [21] для произвольной размерности пространства $N \geqslant 1$. Существование стоячих волн изучалось в [22], [23]. Разрушение и глобальное существование решений исследовалось в [24], [25].

В настоящей работе изучается класс квазилинейных уравнений Шрёдингера вида

$$
i \varphi_{t}=-\Delta \varphi-|\varphi|^{p-1} \varphi-\Delta\left(|\varphi|^{2}\right) \varphi, \quad t \geqslant 0, x \in \mathbb{R}^{N}
$$

здесь $\varphi=\varphi(t, x): \mathbb{R}^{+} \times \mathbb{R}^{N} \rightarrow \mathbb{C}, N$ - размерность пространства, $i=\sqrt{-1}$, $\Delta$ - оператор Лапласа, а $p$ лежит в интервале $\left(1,(N+2) /(N-2)^{+}\right)$, где

$$
\frac{N+2}{(N-2)^{+}}= \begin{cases}(N+2) /(N-2) & \text { при } N \geqslant 3, \\ \infty & \text { при } N=1,2 .\end{cases}
$$

Уравнение (3) описывает распространение верхнегибридных колебаний в физике $([3],[11])$.

*Работа частично поддержана ГФЕН (проекты 10747148 и 10771151) и Научноисследовательским фондом Департамента образования провинции Сычуань. 
Данная статья посвящена изучению точного условия глобального существования решений задачи Коши для уравнения (3). Мы вводим вариационную задачу с перекрестными ограничениями и так называемые инвариантные множества и получаем некоторые новые теоремы о разрушении решений и точный критерий глобального существования или разрушения решений уравнения (3). Такие рассуждения восходят к работам Берестицкого и Казенава [26], Вайнстейна [27], Чжана [28], [29], Пэйна и Сэттингера [30], Ливайна [31].

Работа устроена следующим образом. Некоторые предварительные сведения содержатся в $\S 2$. В 33 строится вариационная задача с перекрестными ограничениями. В $\S 4$ доказывается точное условие разрушения или глобального существования решений.

\section{§2. Предварительные сведения}

Зададим начальные данные для уравнения (3) в виде

$$
\varphi(0, x)=\varphi_{0}, \quad x \in \mathbb{R}^{N},
$$

и определим энергетическое пространство формулой

$$
H_{r}^{1}\left(\mathbb{R}^{N}\right)=\left\{u \in H^{1}\left(\mathbb{R}^{N}\right): u(x)=u(|x|)\right\} .
$$

Здесь и далее для простоты интеграл $\int_{\mathbb{R}^{N}} \ldots d x$ обозначается через $\int \ldots d x$.

Сформулируем две леммы (см. [32], [33]).

Лемма 2.1. Пусть $\varphi_{0} \in H_{r}^{1}\left(\mathbb{R}^{N}\right)$, и пусть $\varphi$ - решение задачи Коши (3)(4) в пространстве $C\left([0, T) ; H_{r}^{1}\left(\mathbb{R}^{N}\right)\right)$. Рассмотрим функционал энергии

$$
E(t)=\int\left(|\nabla \varphi|^{2}+\left.\left.\frac{1}{2}|\nabla| \varphi\right|^{2}\right|^{2}-\frac{2}{p+1}|\varphi|^{p+1}\right) d x .
$$

Тогда

$$
\begin{aligned}
\int|\varphi|^{2} d x & =\int\left|\varphi_{0}\right|^{2} d x, \\
E(t) & \equiv E(0) .
\end{aligned}
$$

Лемма 2.2. Пусть $\varphi_{0} \in H_{r}^{1}\left(\mathbb{R}^{N}\right)$, и пусть $\varphi$ - решение задачи Коши (3)-(4) в пространстве $C\left([0, T) ; H_{r}^{1}\left(\mathbb{R}^{N}\right)\right)$, a $|x| \varphi_{0} \in L^{2}\left(\mathbb{R}^{N}\right)$. Положим $J(t)=$ $\int|x|^{2}|\varphi|^{2} d x$. Тогда

$$
J^{\prime \prime}(t)=8 \int\left(|\nabla \varphi|^{2}+\left.\left.\frac{N+2}{4}|\nabla| \varphi\right|^{2}\right|^{2}-\frac{N(p-1)}{2(p+1)}|\varphi|^{p+1}\right) d x .
$$

\section{§3. Вариационная задача с перекрестными ограничениями}

Для $u \in H_{r}^{1}\left(\mathbb{R}^{N}\right), \omega>0$ зададим функционалы

$$
\begin{aligned}
I(u) & =\frac{1}{2} \int\left(|\nabla u|^{2}+\omega|u|^{2}+\left.\left.\frac{1}{2}|\nabla| u\right|^{2}\right|^{2}-\frac{2}{p+1}|u|^{p+1}\right) d x, \\
S(u) & =\int\left(|\nabla u|^{2}+\omega|u|^{2}+\left.\left.\frac{1}{2}|\nabla| u\right|^{2}\right|^{2}-|u|^{p+1}\right) d x, \\
Q(u) & =\int\left(|\nabla u|^{2}+\left.\left.\frac{N+2}{4}|\nabla| u\right|^{2}\right|^{2}-\frac{N(p-1)}{2(p+1)}|u|^{p+1}\right) d x
\end{aligned}
$$


и определим множество

$$
M:=\left\{u \in H_{r}^{1}\left(\mathbb{R}^{N}\right) \backslash\{0\}, S(u)<0, Q(u)=0\right\} .
$$

Рассмотрим следующие две вариационные задачи с ограничениями:

$$
\begin{aligned}
d_{\omega} & =\inf _{u \in H_{r}^{1}\left(\mathbb{R}^{N}\right) \backslash\{0\}, S(u)=0} I(u), \\
d_{M} & =\inf _{u \in M} I(u) .
\end{aligned}
$$

Прежде всего из (11) получается следующее утверждение.

Лемма 3.1. $d_{\omega}>0$.

Доказательство. Поскольку $S(u)=0$, из теоремы вложения Соболева получаем

$$
\begin{aligned}
\int|u|^{p+1} d x & \leqslant c\left(\int\left(|\nabla u|^{2}+\omega|u|^{2}\right) d x\right)^{(p+1) / 2} \\
& \leqslant c\left[\int\left(|\nabla u|^{2}+\omega|u|^{2}+\left.\left.\frac{1}{2}|\nabla| u\right|^{2}\right|^{2}\right) d x\right]^{(p+1) / 2} \\
& =c\left(\int|u|^{p+1} d x\right)^{(p+1) / 2} .
\end{aligned}
$$

Здесь и далее буква $c$ обозначает различные положительные постоянные. Поскольку $p>1$, а $u \neq 0$, из неравенства (13) следует, что

$$
\int|u|^{p+1} d x \geqslant c>0 .
$$

В силу (11) имеем

$$
I(u)=\frac{p-1}{2(p+1)} \int|u|^{p+1} d x .
$$

Таким образом, $d_{\omega} \geqslant c>0$.

Далее, из (12) вытекает следующее утверждение.

Лемма 3.2. Если $3+4 / N<p<(N+2) /(N-2)^{+}$, mo $_{M}>0$.

Доказательство. Пусть $u \in M$. Из неравенства $S(u)<0$ вытекает, что $u \neq 0$. Так как $Q(u)=0$, то

$$
I(u)=\int\left(\left(\frac{1}{2}-\frac{2}{N(p-1)}\right)|\nabla u|^{2}+\frac{1}{2} \omega|u|^{2}+\left.\left.\left(\frac{1}{8}-\frac{N+2}{4 N(p-1)}\right)|\nabla| u\right|^{2}\right|^{2}\right) d x .
$$

Поскольку $3+4 / N<p<(N+2) /(N-2)^{+}$, из формулы (16) и соотношения $u \neq 0$ следует, что $I(u)>0$ для всех $u \in M$. Таким образом, из (12) получаем, что $d_{M} \geqslant 0$. Воспользуемся теперь неравенством из теоремы вложения Соболева:

$$
\int|u|^{p+1} d x \leqslant c\left(\int\left(|\nabla u|^{2}+\omega|u|^{2}\right) d x\right)^{(p+1) / 2} .
$$


Здесь и далее буква $c$ по-прежнему обозначает различные положительные постоянные. Так как $S(u)<0$, то

$$
\begin{aligned}
\int\left(|\nabla u|^{2}+\omega|u|^{2}+\left.\left.\frac{1}{2}|\nabla| u\right|^{2}\right|^{2}\right) d x & <\int|u|^{p+1} d x \\
& \leqslant c\left(\int\left(|\nabla u|^{2}+\omega|u|^{2}+\left.\left.\frac{1}{2}|\nabla| u\right|^{2}\right|^{2}\right) d x\right)^{(p+1) / 2} .
\end{aligned}
$$

Из неравенства $p>1$ вытекает, что

$$
\int\left(|\nabla u|^{2}+\omega|u|^{2}+|x|^{2}|u|^{2}\right) d x \geqslant c>0 .
$$

Поскольку $p>3+4 / N$, из соотношений (16) и (18) следует, что

$$
I(u) \geqslant c>0 \text { для всех } u \in M .
$$

Итак, $d_{M}>0$ в задаче $(12)$ при $3+4 / N<p<(N+2) /(N-2)^{+}$. Лемма доказана.

Теперь положим

$$
d:=\min \left\{d_{\omega}, d_{M}\right\}
$$

Тогда из лемм 3.1 и 3.2 вытекает следующее утверждение.

Теорема 3.3. При $3+4 / N<p<(N+2) /(N-2)^{+}$имеет место неравенство $d>0$.

Замечание 3.4. Мы называем вариационную задачу (12) вариационной задачей с перекрестными ограничениями, ибо в (12) два условия-ограничения. Соответствующее инвариантное многообразие, определяемое ниже, называется перекрестно-инвариантным многообразием.

Теорема 3.5. Пусть

$$
K:=\left\{\phi \in H_{r}^{1}\left(\mathbb{R}^{N}\right), I(\phi)<d, Q(\phi)<0, S(\phi)<0\right\} .
$$

Тогда множество $K$ является инвариантным многообразием уравнения $(3) ; a$ именно, если $\varphi_{0} \in K$, то решение $\varphi(t, x)$ задачи Коши (3)-(4) лежит в K для любого $t \in[0, T)$.

Доказательство. Пусть $\varphi_{0} \in K$. Существует единственная функция $\varphi(t, x) \in$ $C\left([0, T] ; H_{r}^{1}\left(\mathbb{R}^{N}\right)\right)$, где $T<\infty$, такая, что $\varphi(t, x)$ - решение задачи Коши $(3)-(4)$. Из соотношений (5), (6) вытекает, что

$$
I(\varphi)=I\left(\varphi_{0}\right), \quad t \in[0, T) .
$$

Итак, из неравенства $I\left(\varphi_{0}\right)<d$ следует неравенство $I(\varphi)<d$ для любого $t \in$ $[0, T)$.

Покажем теперь, что $S(\varphi)<0$ при $t \in[0, T)$. Действительно, в противном случае в силу непрерывности существовало бы такое число $t_{0} \in[0, T)$, что $S\left(\varphi\left(t_{0}, \cdot\right)\right)=0$. Согласно $(21)$, имеем $\varphi\left(t_{0}, x\right) \neq 0$. Далее, из (11) и (19) следует, что $I\left(\varphi\left(t_{0}, \cdot\right)\right) \geqslant d$. Но это противоречит неравенству $I(\varphi(t, \cdot))<d$, которое справедливо при $t \in[0, T)$. Следовательно, $S(\varphi(t, \cdot))<0$ для всех $t \in[0, T)$.

Докажем, наконец, что $Q(\varphi(t, \cdot))<0$ при $t \in[0, T)$. Действительно, иначе в силу непрерывности нашлось бы такое число $t_{1} \in[0, T)$, что $Q\left(\varphi\left(t_{1}, \cdot\right)\right)=0$. Мы уже показали, что $S\left(\varphi\left(t_{1}, \cdot\right)\right)<0$, так что $\varphi\left(t_{1}, \cdot\right) \in M$. Итак, из формул (11) и (19) следует, что $I\left(\varphi\left(t_{1}, \cdot\right)\right) \geqslant d_{M} \geqslant d$. Но это противоречит неравенству 
$I(\varphi(t, \cdot))<d$, которое выполнено при $t \in[0, T)$. Поэтому $Q(\varphi(t, \cdot))<0$ при всех $t \in[0, T)$.

Итак, в силу предыдущего мы доказали, что $\varphi(t, x) \in K$ для любого $t \in[0, T)$.

Доказательство теоремы завершено.

Рассуждая так же, как и в теореме 3.5 , можно получить следующие результаты.

Теорема 3.6. Положим

$$
\begin{aligned}
& K_{+}:=\left\{\phi \in H_{r}^{1}\left(\mathbb{R}^{N}\right), I(\phi)<d, Q(\phi)>0, S(\phi)<0\right\}, \\
& R_{-}:=\left\{\phi \in H_{r}^{1}\left(\mathbb{R}^{N}\right), I(\phi)<d, S(\phi)<0\right\}, \\
& R_{+}:=\left\{\phi \in H_{r}^{1}\left(\mathbb{R}^{N}\right), I(\phi)<d, S(\phi)>0\right\} .
\end{aligned}
$$

Тогда $K_{+}, R_{-}$и $R_{+}-$инвариантнъе многообразия задачи (3)-(4).

\section{§4. Точные условия глобального существования решений}

Теорема 4.1. Пусть $3+4 / N<p<(N+2) /(N-2)^{+}$. Если $\varphi_{0} \in K_{+} \cup R_{+}$, то решение $\varphi$ задачи Коши (3)-(4) существует глобально при $t \in[0, \infty)$.

Доказательство. Предположим сначала, что $\varphi_{0} \in K_{+}$. Тогда из теоремы 3.6 вытекает, что для решения $\varphi$ задачи Коши (3)-(4) справедливо включение $\varphi(t, \cdot) \in K_{+}$при $t \in[0, T)$. Для заданного $t \in[0, T)$ положим $\varphi(t, \cdot)=\varphi$. Итак, $I(\varphi)<d$ и $Q(\varphi)>0$. Из формул (8) и (10) следует, что

$$
\int\left(\left(\frac{1}{2}-\frac{2}{N(p-1)}\right)|\nabla \varphi|^{2}+\frac{1}{2} \omega|\varphi|^{2}+\left.\left.\left(\frac{1}{8}-\frac{N+2}{4 N(p-1)}\right)|\nabla| \varphi\right|^{2}\right|^{2}\right) d x<d .
$$

Значит,

$$
\int|\nabla \varphi|^{2} d x<c
$$

Отсюда вытекает, что решение $\varphi$ существует глобально на интервале $t \in[0, \infty)$.

Итак, мы доказали, что при $\varphi_{0} \in K_{+}$решение $\varphi$ задачи Коши (1) существует глобально на интервале $t \in[0, \infty)$.

Пусть теперь $\varphi_{0} \in R_{+}$. Тогда из теоремы 3.6 следует, что для решения $\varphi$ задачи Коши (1) справедливо включение $\varphi(t, \cdot) \in R_{+}$при всех $t \in[0, T)$. Таким образом, $I(\varphi)<d$ и $S(\varphi)>0$. Отсюда вытекает, что

$$
\left(\frac{1}{2}-\frac{1}{p+1}\right) \int\left(|\nabla \varphi|^{2}+\omega|\varphi|^{2}+\left.\left.\frac{1}{2}|\nabla| \varphi\right|^{2}\right|^{2}\right) d x<d .
$$

Следовательно, решение $\varphi$ существует глобально на интервале $t \in[0, \infty)$.

Теорема доказана.

Теорема 4.2. Пусть $3+4 / N<p<(N+2) /(N-2)^{+}$. Если $\varphi_{0} \in K u$ $|x| \varphi_{0} \in L^{2}\left(\mathbb{R}^{N}\right)$, то решение $\varphi$ задачи Коши (3)-(4) разрушается за конечное время.

Доказательство. Поскольку $\varphi_{0} \in K$, из теоремы 3.5 вытекает, что для решения $\varphi$ задачи Коши (3)-(4) при $t \in[0, T)$ справедливо включение $\varphi(t, \cdot) \in K$. Для функции $J(t)=\int|x|^{2}|\varphi|^{2} d x$ из (7) и (10) вытекает равенство

$$
J^{\prime \prime}(t)=8 Q(\varphi(t, \cdot)), \quad t \in[0, T) .
$$


Заметим, что так как $Q(\varphi)<0$, то

$$
J^{\prime \prime}(t)<0 .
$$

Очевидно, что функция $J(t)$ не может удовлетворять неравенству (26) для всех $t$ (см. [33]). Поэтому непременно существует такое число $T<\infty$, что

$$
\lim _{t \rightarrow T}\|\varphi\|_{H_{r}^{1}\left(\mathbb{R}^{N}\right)}=\infty .
$$

Теорема доказана.

Замечание 4.3. Ясно, что

$$
\left\{\phi \in H_{r}^{1}\left(\mathbb{R}^{N}\right), I(\phi)<d\right\}=R_{+} \cup K_{+} \cup K .
$$

Таким образом, теорема 4.2 показывает, что теорема 4.1 не может быть улучшена.

\section{ЛиТЕРАТУРА}

[1] S. Kurihura, Large-amplitude quasi-solitons in superfluid films, J. Phys. Soc. Japan, 50:10 (1981), 3262-3267.

[2] H. Lange, M. Poppenberg, H. Teismann, Nash-Moser methods for the solution of quasilinear Schrödinger equations, Comm. Partial Differential Equations, 24:7-8 (1999), 1399-1418.

[3] E. W. Laedke, K. H. Spatschek, L. Stenflo, Evolution theorem for a class of perturbed envelope soliton solutions, J. Math. Phys., 24:12 (1983), 2764-2769.

[4] А. В. Боровский, А. Л. Галкин, Динамическая модулячия ультракороткого интенсивного лазерного импульса в веществе, ЖЭТФ, 104:10 (1993), 3311-3333.

[5] H. S. Brandi, C. Manus, G. Mainfray, T. Lehner, G. Bonnaud, Relativistic and ponderomotive self-focusing of a laser beam in a radially inhomogeneous plasma, Phys. Fluids B, 5 (1993), 3539-3550.

[6] X. L. Chen, R. N. Sudan, Necessary and sufficient conditions for self-focusing of short ultraintense laser pulse in underdence plasma, Phys. Rev. Lett., 70:14 (1993), 20822085.

[7] B. Ritchie, Relativistic self-focusing and channel formation in laser-plasma interactions, Phys. Rev. E, 50:2 (1994), 687-689.

[8] A. De Bouard, N. Hayashi, J. C. Saut, Global existence of small solutions to a relativistic nonlinear Schrödinger equation, Comm. Math. Phys., 189:1 (1997), 73-105.

[9] А. Г. Литвак, А. М. Сергеев, Об одномерном коллапсе плазменных волн, Письма в ЖЭТФ, 27:10 (1978), 549-553.

[10] A. Nakamura, Damping and modification of exciton solitary waves, J. Phys. Soc. Japan, 42:6 (1977), 1824-1835.

[11] M. Porkolab, M. V. Goddman, Upper-hybrid solitons and oscillating-two-stream instabilities, Phys. Fluids, 19:6 (1976), 872-881.

[12] F. G. Bass, N. N. Nasanov, Nonlinear electromagnetic spin waves, Phys. Rep., 189:4 (1990), 165-223.

[13] A. M. Kosevich, B. A. Ivanov, A. S. Kovalev, Magnetic solitons, Phys. Rep., 194:3-4 (1990), 117-238.

[14] H. Lange, B. Toomire, P. E. Zweifel, Time-dependent dissipation in nonlinear Schrödinger systems, J. Math. Phys., 36:3 (1995), 1274-1283.

[15] G. R. W. Quispel, H. W. Capel, Equation of motion for the Heisenberg spin chain, Physica A, 110:1-2 (1982), 41-80.

[16] S. Takeno, S. Homma, Classical planar Heisenberg ferromagnet, complex scalar fields and nonlinear excitations, Progr. Theoret. Phys., 65:1 (1981), 172-189. 
[17] R. W. Hasse, A general method for the solution of nonlinear soliton and kink Schrödinger equations, Z. Phys. B, 37:1 (1980), 83-87.

[18] V. G. Makhankov, V. K. Fedyanin, Non-linear effects in quasi-one-dimensional models of condensed matter theory, Phys. Rep., 104:1 (1984), 1-86.

[19] M. Poppenberg, Smooth solutions for a class of fully nonlinear Schrödinger type equations, Nonlinear Anal., 45:6 (2001), 723-741.

[20] M. Poppenberg, On the local well posedness of quasi-linear Schrödinger equations in arbitrary space dimension, J. Differential Equations, 172:1 (2001), 83-115.

[21] M. Poppenberg, An inverse function theorem in Sobolev spaces and applications to quasi-linear Schrödinger equations, J. Math. Anal. Appl., 258:1 (2001), 146-170.

[22] M. Poppenberg, K. Schmitt, Z. Q. Wang, On the existence of soliton solutions to quasilinear Schrödinger equations, Calc. Var. Partial Differential Equations, 14:3 (2002), 329-344.

[23] J. Q. Liu, Y. Q. Wang, Z. Q. Wang, Soliton solutions for quasi-linear Schrödinger equations II, J. Differential Equations, 187:2 (2003), 473-493.

[24] J. J. García-Ripoll, V. V. Konotop, B. Malomed, V. M. Pérez-García, A quasi-local Gross-Pitaevskii equation for attractive Bose-Einstein condensates, Math. Comput. Simulation, 62:1-2 (2003), 21-30.

[25] J. Zhang, The blowup properties of the initial-boundary problem for second order derivative nonlinear Schrödinger equations, Acta Math. Sci., 14 (1994), 89-94.

[26] H. Berestycki, T. Cazenave, Instabilité des états stationnaires dans les équations de Schrödinger et de Klein-Gordon non linéarires, C. R. Acad. Sci. Paris, Seire I, 293 (1981), 489-492.

[27] M. I. Weinstein, Nonlinear Schrödinger equations and sharp interpolations estimates, Comm. Math. Phys., 87 (1983), 567-576.

[28] J. Zhang, Sharp conditions of global existence for nonlinear Schrödinger and KleinGordon equations, Nonlinear Anal., 48:2 (2002), 191-207.

[29] J. Zhang, Sharp threshold of blowup and global existence in nonlinear Schrödinger equations under a harmonic potential, Comm. Partial Differential Equations, 30:1012 (2005), 1429-1443.

[30] L. E. Payne, D. H. Sattinger, Saddle points and instability of nonlinear hyperbolic equations, Israel J. Math., 22:3-4 (1975), 273-303.

[31] H. A. Levine, Instability and non-existence of global solutions to nonlinear wave equations of the form $P u_{t t}=-A u+F(u)$, Trans. Amer. Math. Soc, 192 (1974), 1-21.

[32] T. Cazenave, An introduction to nonlinear Schrödinger equations, Textos de Metodos Mathematicos, Rio de Janeiro, vol. 26, 1993.

[33] Y. Tsutsumi, J. Zhang, Instability of optical solitons for two-wave interaction model in cubic nonlinear media, Adv. Math. Sci. Appl., 8:2 (1998), 691-713.

Ведущая лаборатория компьютерных программ провинции Сычуань Сычуаньского нормального университета

Колледж математики и программирования Сычуаньского нормального университета

Колледж математики и программирования

Сычуаньского нормального университета

Ведущая лаборатория компьютерных программ провинции Сычуань Сычуаньского нормального университета

e-mails: shu.ji@163.com; shuji2008@hotmail.com
Поступило в редакцию 7 декабря 2005 г.

В переработанном виде 23 декабря 2007 г. 